\title{
Third flow component as QGP signal
}

\author{
L.P. Csernai ${ }^{\mathrm{a}, \mathrm{b}, 1}$ and D. Röhrich ${ }^{\mathrm{a}}$ \\ ${ }^{a}$ Department of Physics, University of Bergen \\ Allégaten 55, 5007 Bergen, Norway \\ ${ }^{\mathrm{b}}$ KFKI - Research Inst. for Particle and Nuclear Physics \\ P.O.Box 49, 1525 Budapest 114, Hungary
}

\begin{abstract}
A review of earlier fluid dynamical calculations with QGP show a softening of the directed flow while with hadronic matter this effect is absent. The effect shows up in the reaction plane as enhanced emission which is orthogonal to the directed flow. Thus, it is not shadowed by the deflected projectile and target. As both of these flow components are in the reaction plane these form an enhanced 'elliptic flow' pattern. Recent experimental data at $11 \mathrm{AGeV}$ and above show the same softening, hinting at QGP formation.
\end{abstract}

\section{Introduction}

It is widely known that fluid dynamics (FD) is governed by the Equation of State (EoS) of the matter, and the analysis of the resulting flow patterns turned out to be one of the best tools to extract the EoS from the outcome of a heavy ion collision. The final event shape must carry the information about the pressure development during the collision including the early stages of the collision.

The phase transition to the Quark-Gluon Plasma (QGP) is connected to a decrease in pressure according to most theoretical estimates, not only in strong first order phase transition models, but even if we have a smooth but rapid gradual transition. This reduced pressure and temperature around the phase transition threshold is known for long (see e.g. [1]), and it is emphasized recently as a QGP signal, i.e., the "soft point" of the EoS, which should be

1 Partially supported by Research Council of Norway (NFR) and by the Alexander von Humboldt Foundation. 
possible to observe in excitation functions of collective flow data[5,6]. It was pointed out earlier [3] that the decrease in the out of plane (squeeze out) emission is even more sensitive to the pressure drop and it decreases due to plasma formation stronger than the inplane collective flow.

Here we want to point out another consequence of the same softening in the EoS, which is a new distinct flow pattern, which can be seen in almost all theoretical FD calculations with QGP formation, but which was not discussed earlier $[2-5,7]$.

\section{Third flow component in fluid dynamics}

Up to now two basic flow patterns are predicted and detected: (i) the directed transverse flow in the reaction plane, or side-splash, or bounce off, which is most frequently presented on the well known $P_{x}$ vs. $y$ diagram and seen at all energies in heavy ion collisions from energies of $30 \mathrm{~A} . \mathrm{MeV}$ to $165 \mathrm{~A} . \mathrm{GeV}$, $[10,13,11,12]$ and the (ii) squeeze-out effect which is an enhanced emission of particles transverse to the reaction plane at center of mass (CM) rapidities.

At lower energies the directed transverse flow resulted in a smooth, linear $P_{x}$ vs. $y$ dependence at CM rapidities. This straight line behavior connecting the maximum at $y_{\text {proj }}$ and the minimum at $y_{\text {targ }}$ was so typical that it was used to compare flow data at different beam energies and masses.

If QGP is formed, strong and rapid equilibration and stopping takes place, and close to one-fluid behavior is established. Stopping is stronger than expected, and Landau's fluid dynamical model is becoming applicable for central collisions of massive heavy ions. The soft and compressible QGP forms a rather flat disk orthogonal to the beam axis which is at rest in the CM system. Then this disk starts to expand rapidly in the direction of the largest pressure gradient, i.e., forward and backward. Thus, the not fully Gaussian shape of the measured rapidity spectra can be interpreted as a fluid dynamical bounce back effect (Landau model) in contrast to the transparency otherwise assumed in kinetic models. Unfortunately in central collisions we can not distinguish the two effects from one - another. Both lead to a spectrum elongated in the beam direction.

At small but finite impact parameters, however, this disk is tilted, and the direction of fastest expansion, will deviate from the beam axis, will stay in the reaction plane, but point in directions opposite to the standard directed transverse flow. Since pressure does not play a role in transparency, transparency cannot explain such deviation from the beam direction! This third flow com- 
ponent develops purely from the large pressure gradient at full stopping of the strongly Lorentz contracted intermediate state. So, at the same time as the primary directed flow is weakened by the stronger Lorentz contraction at higher energies, this third flow component is strengthened by increased Lorentz contraction. These two flow components together form the 'elliptic flow'.[11,16,17]

On the $P_{x}$ vs. $y$ diagram [14] this component shows up as a smaller, negative flow component at small CM rapidities. Such a third flow component is seen clearly in Fig. 3 of [3] (see Fig. 1, lower part), Fig. 8 of [4], Fig. 6b of [5] and Fig. 6 of [7] at or slightly below $0.5 \mathrm{y} / y_{\mathrm{cm}}$ if QGP formation was allowed during the calculation. In sharp contrast, the solutions with hadronic EoS did not show this effect, and the maximum and minimum of the $P_{x}$ curve could be connected with a rather straight line. This straight line behavior is typical at all flow results below $11 \mathrm{~A} . \mathrm{GeV}$ beam energy (Fig. 2). In some of the FD calculations with QGP the secondary peak at small CM rapidities is not seen, but a tendency is obvious, and the deviation from the hadronic smooth line behavior is apparent. This can be seen clearly in Fig. 3 of [2], and Figs. $6 \mathrm{a}$ and $6 \mathrm{c}$ of [5]. This indicates that the strength of this effect is also impact parameter and beam energy dependent, and the third flow component shows a relative maximum at the same energy when the primary directed flow is at its minimum[5]. Note that all these FD calculations were done much before the experiments. The first quantitative flow predictions [2] preceded the experiments by as much as 6 years (!) and gave rather good agreements with the data.

To have a quantitative measure of the softening at small $\mathrm{CM}$ rapidities $\left(y_{C M}=\right.$ 0 ) for a symmetric $(\mathrm{A}+\mathrm{A})$ collision, on the $\left[P_{x}, y\right]$ plane we draw the steepest straight line, ay, through the CM point which is tangent to the $P_{x}(y)$ curve (Fig. 1 upper part). The $P_{x}(y)$ curve and the straight line are usually tangent to each other at $y=0$, and at finite $y$ the $P_{x}(y)$ curve is under the straight line, $|a y|>\left|P_{x}(y)\right|$. In case of softening at low rapidities the straight line is tangent to the $P_{x}(y)$ curve at two points, $y_{1}$ and $-y_{1}$ and at smaller rapidities, and at smaller but finite rapidities, $0<|y|<\left|y_{1}\right|$, the $P_{x}(y)$ curve is under the straight line, $|a y|>\left|P_{x}(y)\right|$. The relative deviation of these two quantities, $S(y) \equiv\left|a y-P_{x}(y)\right| /|a y|$ has a maximum at some rapidity, $0<\left|y=y_{\max }\right|<$ $\left|y_{1}\right|$, and we will use this quantity, $S\left(y_{\max }\right)$, to characterize the softening of EoS. If $S=100 \%$ this means that $P_{x}(y)$ vanishes somewhere between 0 and $\left|y_{1}\right|$. If $S>100 \%$ this means that $P_{x}$ is inverted (shows negative flow) at low rapidities.

Thus in FD calculations, this effect is clearly predicted since 1991, in all calculations, without being noticed or discussed up to now. Nevertheless, all FD calculations are consistent in predicting an observable softening if and only if 


\begin{tabular}{|c|c|c|}
\hline Publication & QGP & $\mathrm{HM}$ \\
\hline$[5] \mathrm{Au}+\mathrm{Au} 3.5 \mathrm{~A} \cdot \mathrm{GeV} b=3 \mathrm{fm}$ & $40 \%$ & $0 \%$ \\
\hline$[5] \mathrm{Au}+\mathrm{Au} 5 \mathrm{~A} \cdot \mathrm{GeV} b=3 \mathrm{fm}$ (soft pt.) & $160 \pm 30 \%$ & $0 \pm 20 \%$ \\
\hline$[5] \mathrm{Au}+\mathrm{Au} 11.7 \mathrm{~A} \cdot \mathrm{GeV} b=3 \mathrm{fm}$ & $70 \pm 10 \%$ & $30 \pm 30 \%$ \\
\hline$[3] \mathrm{Au}+\mathrm{Au} 11.6 \mathrm{~A} \cdot \mathrm{GeV} b=5 \mathrm{fm}$ & $100 \pm 10 \%$ & $0 \%$ \\
\hline [4] $\mathrm{Au}+\mathrm{Au} 11.6 \mathrm{~A} \cdot \mathrm{GeV} b=0-0.5 b_{\max }$ & $180 \pm 25 \%$ & $30 \pm 30 \%$ \\
\hline [7] $\mathrm{Au}+\mathrm{Au} 11 \mathrm{~A} \cdot \mathrm{GeV} b=3 \mathrm{fm} 6 \mathrm{fm} / \mathrm{c}$ & $70 \pm 30 \%$ & - \\
\hline [7] $\mathrm{Au}+\mathrm{Au} 11 \mathrm{~A} \cdot \mathrm{GeV} b=3 \mathrm{fm} 7.2 \mathrm{fm} / \mathrm{c}$ & $50 \pm 35 \%$ & - \\
\hline$[2] \mathrm{Pb}+\mathrm{Pb} 160 \mathrm{~A} \cdot \mathrm{GeV} b=4 \mathrm{fm} 3.8 \mathrm{fm} / \mathrm{c}$ & $80 \pm 10 \%$ & $50 \pm 45 \%$ \\
\hline
\end{tabular}

Table 1

Softening in fluid dynamical model calculations which included the possibility of QGP formation in the EoS assumed. When Hadronic Matter (HM) EoS was also presented in the same calculation the resulting softening is also shown for this case. All HM calculations are consistent with zero softening, while calculations with QGP show softening values from 50 to 180\%. (In [7] the non-equilibrium 3-fluid hydro is applied with complex EoS, i.e. hadron gas EoS for the two baryon fluids (no QGP), and EoS with QGP-hadron phase transition, which is used only for the third, baryonfree fluid. Although the effects like directed flow are provided by baryons the flow effect arises from the pressure of the central baryon free component. Nevertheless, non-equilibrium effects in 3-fluid hydrodynamical models could mimic the effect caused by the QGP in ideal 1-fluid models, and so the comparison of this model with other fluid dynamical models is somewhat arbitrary.)

QGP EoS is included in the calculation.

\section{Deviation from straight line behavior in string models}

Some of the recent flow data are analyzed by the Fourier expansion method $[15,16]$. Unfortunately, it is not possible to convert or compare these data to the earlier $P_{x}$ analysis, but the results indicate that the $v_{1}$ vs. $y / y_{c m}$ has a straight line behavior at low rapidities in the absence of QGP (or softening) in the EoS. Thus, just as in the $P_{x}$ analysis we consider that deviation from this straight line behavior indicates a stronger contraction and softening of the EoS. To make the comparison more quantitative would only be possible if data showing such deviation from the straight line behavior would be analyzed by both methods simultaneously. Nevertheless, heavy ion collisions at lower energies, around $2 \mathrm{~A} . \mathrm{GeV}$ show straight line behavior according to both analyses[19]. Furthermore, small relative deviations from the straight line be- 
havior (or softening) are expected to be identical in the two analyses [10] since $\left\langle P_{x}\right\rangle=\left\langle v_{1}\right\rangle \cdot\left\langle P_{t}\right\rangle$.

We do not intend to compare FD models and non-equilibrium models like string models as theoretically they are based on different fundations. However, we review how these models describe the data. In the last decade string models showed a surprising level of resilience by being able to reproduce more or less all measured data. Unlike with FD models this always happened after the data became known. Of course during this time the models were changed essentially and many new ingredients were built in. Some of these changes are in fact rather essential, and change the physical picture described by these models drastically. One of the most important changes is the introduction of string ropes (fused strings, or quark clusters), and only this made it possible to describe the formation of massive formed secondaries like strange antibaryons. Recent flow data are also stretching the string models flexibility to their limits, while FD models as usual overestimate collective flow data.

In Table 2 we present a collection of string model results regarding the low rapidity softening.

In an attempt to fit all data with string models in ref. [8] RQMD was particularly tested to which extent it is able to reproduce NA49 flow data. Fig. 3 [8] presents the $v_{1}$ vs. $y$ plot for $158 \mathrm{~A} . \mathrm{GeV} \mathrm{Pb}+\mathrm{Pb}$ data. The proton data show a very strong deviation from the straight line behavior (indicating strong EoS softening based on analogies with $P_{x}$ results). The best fits with RQMD v 2.3 also reproduce some of the deviation from the straight line behavior, but at low rapidities the model result lags behind the SPS data appreciably, while it yields also some softening at $2 \mathrm{~A} . \mathrm{GeV}$ where experiments show no sign of such behavior.

Venus and to some extent RQMD might include some of the genuine flow effects as the increasing Lorentz contraction, increasing pressure, and increased stopping, but these do not quite reach the extent one can have in an FD model with QGP.

Although, the authors of ref. [8] conclude that in RQMD the deviation from the straight line behavior is caused mainly by shadowing, it is difficult to observe any systematic viewing all published RQMD results. The shadowing effect is also confirmed by an earlier work [9] which actually went more into details, and showed that for heavy systems like $\mathrm{Pb}+\mathrm{Pb}$ the shadowing starts to cause deviations from the straight line behavior only in peripheral reactions $\left(b>0.75 b_{\max }\right)$. For lighter systems the deviation may show up already at smaller impact parameters also. This coincides with the result shown in Fig. 2 of [8], for $\mathrm{Pb}+\mathrm{Pb}$ and $\mathrm{S}+\mathrm{S}$ at $158 \mathrm{~A} \cdot \mathrm{GeV}$. 


\begin{tabular}{|c|c|}
\hline Publication & $\mathrm{S}$ \\
\hline [18] Venus $4.12 \mathrm{~Pb}+\mathrm{Pb} 158 \mathrm{~A} . \mathrm{GeV} v_{1}$ & $110 \pm 15 \%$ \\
\hline [12] Venus $4.12 \mathrm{~Pb}+\mathrm{Pb} 158 \mathrm{~A} \cdot \mathrm{GeV} b=5-12 \mathrm{fm} P_{x}$ & $134 \pm 15 \%$ \\
\hline [8] RQMD 2.3 Pb+Pb 2 A.GeV $b=5-8$ fm Fig. $2 v_{1}$ & $40 \%$ \\
\hline [10] RQMD $2.3 \mathrm{Au}+\mathrm{Au} 11.6 \mathrm{~A} \cdot \mathrm{GeV} P_{x}$ & $32 \pm 20 \%$ \\
\hline [10] RQMD $1.08 \mathrm{Au}+\mathrm{Au} 11.6 \mathrm{~A} . \mathrm{GeV} P_{x}$ & $0 \pm 20 \%$ \\
\hline [12] RQMD 2.3 Pb+Pb $158 \mathrm{~A} \cdot \mathrm{GeV} b=8-10 \mathrm{fm} P_{x}$ & $0 \pm 25 \%$ \\
\hline [8] RQMD $2.3 \mathrm{~Pb}+\mathrm{Pb} 158$ A.GeV Fig. $1 v_{1}$ & $72 \%$ \\
\hline [8] RQMD $2.3 \mathrm{~Pb}+\mathrm{Pb} 158$ A.GeV $b=5-8$ fm Fig. $2 v_{1}$ & $57 \%$ \\
\hline [2] QGSM Pb+Pb 158 A.GeV $b=4 \mathrm{fm} P_{x}$ & $0 \pm 30 \%$ \\
\hline [3] QGSM Au+Au 11.6 A.GeV $b=3 \mathrm{fm} P_{x}$ & $0 \pm 10 \%$ \\
\hline [9] QGSM Au+Au 11.6 A.GeV $b=1, \ldots, 10 \mathrm{fm} P_{x}$ & $0 \%$ \\
\hline [9] QGSM Au+Au 11.6 A.GeV $b=11 \mathrm{fm} P_{x}$ & $35 \%$ \\
\hline [9] QGSM Au+Au $11.6 \mathrm{~A} \cdot \mathrm{GeV} b=12 \mathrm{fm} P_{x}$ & $50 \%$ \\
\hline
\end{tabular}

Table 2

Softening in string model calculations. All QGSM calculations are consistent with zero softening, with the exception of extreme peripheral collisions, where the effects of shadowing are observable. Both Venus 4.12 calculations yield consistently large softening at SPS energies. RQMD results show varying results from zero to $72 \%$.

From these string model results we can conclude that shadowing effects in reality may also result in deviations from the straight line behavior, but in heavy colliding systems this happens only in rather peripheral reactions, which can be excluded experimentally. In the $\mathrm{Pb}+\mathrm{Pb}$ case the shadowing effect below the used impact parameter cut of $b<8 \mathrm{fm}$ is rather weak (cf. [9]) thus cannot be solely responsible for the deviation from the straight line behavior. We have to add that the previously discussed purely fluid dynamical effects also show up in string model results in a weaker form. As this is caused by the considerable softening of the EoS due to string ropes and other massive objects, we can actually observe some level of convergence between FD- and string models. Nevertheless, earlier the authors of string model calculations did not observe the possible fluid dynamical component in the explanation. In a recent version of RQMD the measured flow pattern could be reproduced by including fluid dynamical mechanisms and an EoS.[17]

In view of the extreme variety of string model results, we cannot reach any conclusion whether string models consequently yield low energy softening or not. Furthermore, it is not clear what is the general, physical reason of the softening in those string model calculations where such a softening is present. 


\section{Experimental status}

In connection with the string models we discussed already NA49 data. Other experiments starting at about 2-11 A.GeV (Figs. 2 and 3) show signs of deviation from the straight line behavior. At $11 \mathrm{~A} . \mathrm{GeV}$ and particularly at SPS energies the $P_{x}$ vs. $y$ (Fig. 2 bottom) and the $v_{1}$ vs. $y$ (Fig. 3 bottom) becomes almost flat at or close to CM rapidities. Although the inversion of proton flow is not observed as predicted by some of the FD calculations, the tendency from the straight line behavior is consistently seen in all experiments (Table $3)$.

\begin{tabular}{lr}
\hline Publication & S \\
\hline [13] Au+Au 4 A.GeV $P_{x}$ & $40 \pm 50 \%$ \\
[13] Au+Au 6 A.GeV $P_{x}$ & $10 \pm 35 \%$ \\
[10] Au+Au 11 A.GeV $P_{x}$ protons & $30 \pm 20 \%$ \\
[12] Pb+Pb 158 A.GeV $P_{x}$ semicentr. & $60 \pm 50 \%$ \\
[11] Pb+Pb 158 A.GeV $v_{1}$ & $110 \pm 60 \%$ \\
\hline
\end{tabular}

Table 3

Softening in directed transverse flow data. With increasing beam energy the tendency of an increasing softening is observable. At SPS energies the softening becomes significant. This general trend coincides with the fluid dynamical predictions with QGP EoS.

The quantitative study of this effect in finer impact parameter resolution may even pin down more pronounced deviations in the central rapidity region which is important if we want to use flow data to reconstruct the properties of the underlying EoS.

\section{Conclusion}

The observed flow patterns at ultra-relativistic energies indicate a new flow pattern, which may arise from a highly Lorentz contracted and compressed intermediate state as a consequence of an extremely soft EoS. The presently 
observed deviations from the straight line behavior go beyond hadronic or string model predictions, indicating a softer and more compressed initial or intermediate state than hadronic models can accommodate.

A review of earlier FD calculations which included QGP shows that such an effect was present already in all of them to a smaller or larger extent. This sign of excessive softening of the EoS may indicate that a larger portion of the matter is transformed into a soft phase than assumed in string models. The effect shows up in the reaction plane as enhanced emission which is orthogonal to the directed flow. Thus, it is not shadowed by the deflected projectile and target. [3,9] As both of these flow components are in the reaction plane these form an enhanced 'elliptic flow' pattern.

A review of string model results does not provide us with any clear conclusion about the causes or extent of the softening, although the effect is present in some of the string model calculations.

In order to be more quantitative further and more detailed experiments are needed, as well as further FD calculations would be beneficial. Fluctuations, initial pre-equilibrium transparency, viscosity, etc. may decrease the effect in FD calculations also, thus approaching the real reaction mechanism better than recent calculations.

\section{References}

[1] A. K. Holme, E. F. Staubo, L.P. Csernai, E. Osnes, D. Strottman, Phys. Rev. D40 (1989) 3735.

[2] N. S. Amelin, E.F. Staubo, L.P. Csernai, V.D. Toneev, K.K. Gudima and D.D. Strottman, Phys. Rev. Lett. 67 (1991) 1523.

[3] Fluid Dynamical Predictions for $\mathrm{Au}+\mathrm{Au}$ Collisions at AGS - invited talk L. Bravina, L.P. Csernai, P. Lévai, and D. Strottman, - at the Int. Conf. Heavy-Ion Physics at the AGS: HIPAGS '93, Jan. 13-15, 1993, (MIT Boston, Massachusetts, USA, 1993) p.333.

[4] L. Bravina, L.P. Csernai, P. Lévai and D. Strottman, Phys. Rev. C50 (1994) 2161.

[5] D.H. Rischke, Y. Pürsün, J.A. Maruhn, H. Stöcker and W. Greiner, Heavy Ion Phys. 1 (1995) 309.

[6] C.M. Hung and E.V. Shuryak, Phys. Rev. Lett. 75 (1995) 4003.

[7] J. Brachmann, A. Dumitru, J.A. Maruhn, H. Stöcker, W. Greiner and D. Rischke, Nucl. Phys. A 619 (1997) 391. 
[8] H. Liu, S. Panitkin and N. Xu, (1998) nucl-th/9807021,

[9] L. Bravina, Phys. Lett. B 344 (1995) 49.

[10] J. Barette et al., (E814 Collaboration)) Phys. Rev. Lett. 73 (1994) 2532, Phys. Lett. B 351 (1995) 93, J. Barette et al., (E877 Collaboration)) Phys. Rev.i C 55 (1997) 1420, Phys. Rev. C 56 (1997) 3254.

[11] H. Appelshäuser et al. (NA49 Collaboration), Nucl. Phys. A 638 (1998) 463c, and Phys. Rev. Lett. 80 (1998) 4136.

[12] WA98 Collaboration, M. Kurata et al. and S. Nishimura et al. in Proc. of JaipurICPAQP (1997) and M.M. Aggrawal et al. (WA98 Collaboration), Nucl. Phys. A 638 (1998) 459c, and Phys. Rev. Lett. submitted, (nucl-ex/9807004)

[13] N.N. Ajitanand et al. (E895 Collaboration), Nucl. Phys. A 638 (1997) 415c.

[14] P. Danielevicz and G. Odyniecz, Phys. Lett. B 157 (1985) 146.

[15] J.Y. Ollitraut, Phys. Rev. D 46 (1992) 229.

[16] A.M. Poskanzer and S.A. Voloshin, Phys. Rev. C 58 (1998) 1671.

[17] H. Sorge, Phys. Rev. Lett. 82 (1999) 2048.

[18] A.M. Poskanzer, (NA49) private communication (1998).

[19] E 895 private communication (1998). 


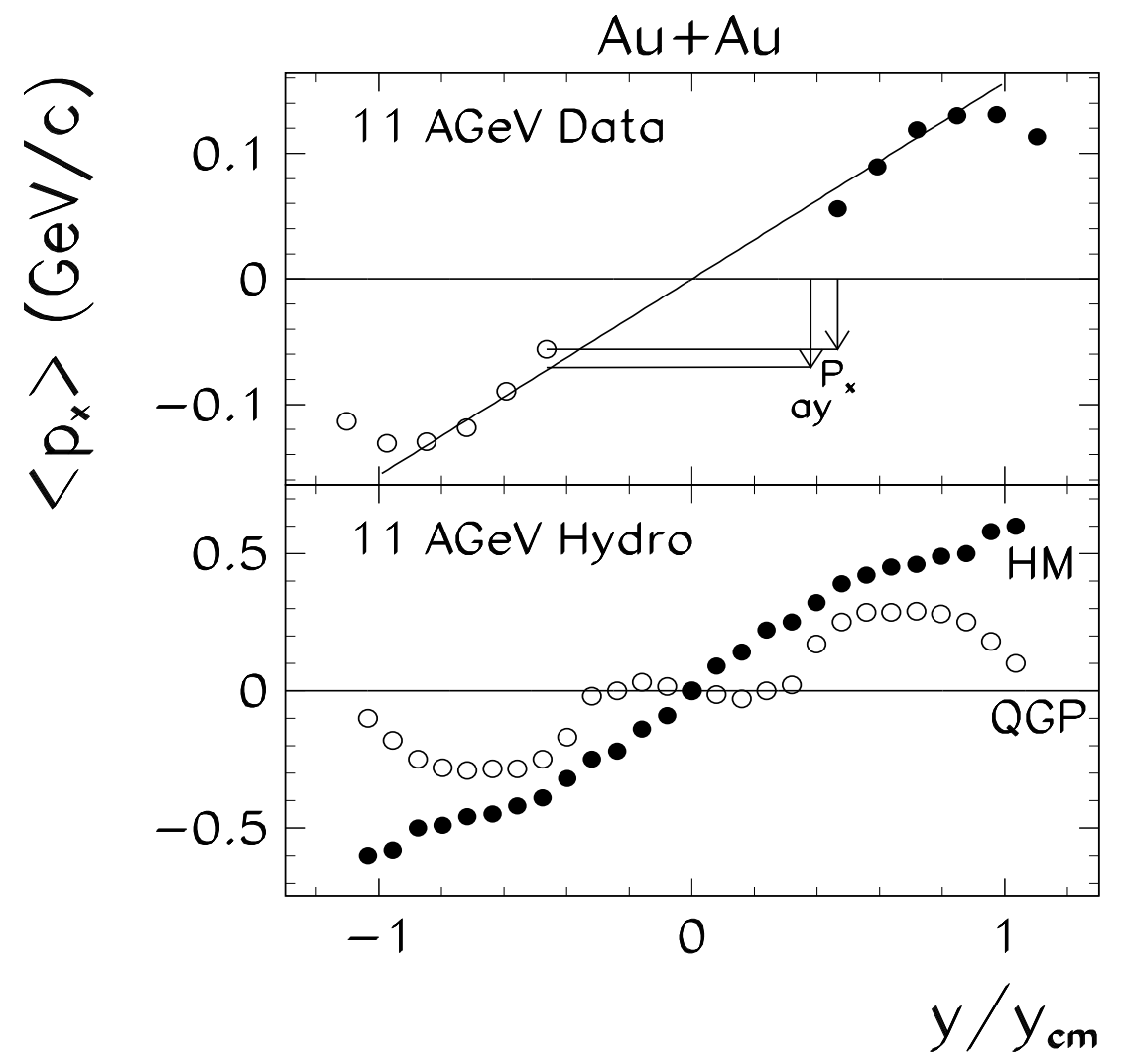

Fig. 1. Upper part: Definition of the measure softening, $S$, describing the deviation of $P_{x}(y)$ or $v_{1}(y)$ from the straight line behavior, ay, around CM. $S$ is defined as $\left|a y-P_{x}(y)\right| /|a y|$. The lower figure shows a typical example for fluid dynamical calculations with Hadronic and QGP EoS [3]. QGP leads to strong softening, $\sim 100 \%$. 


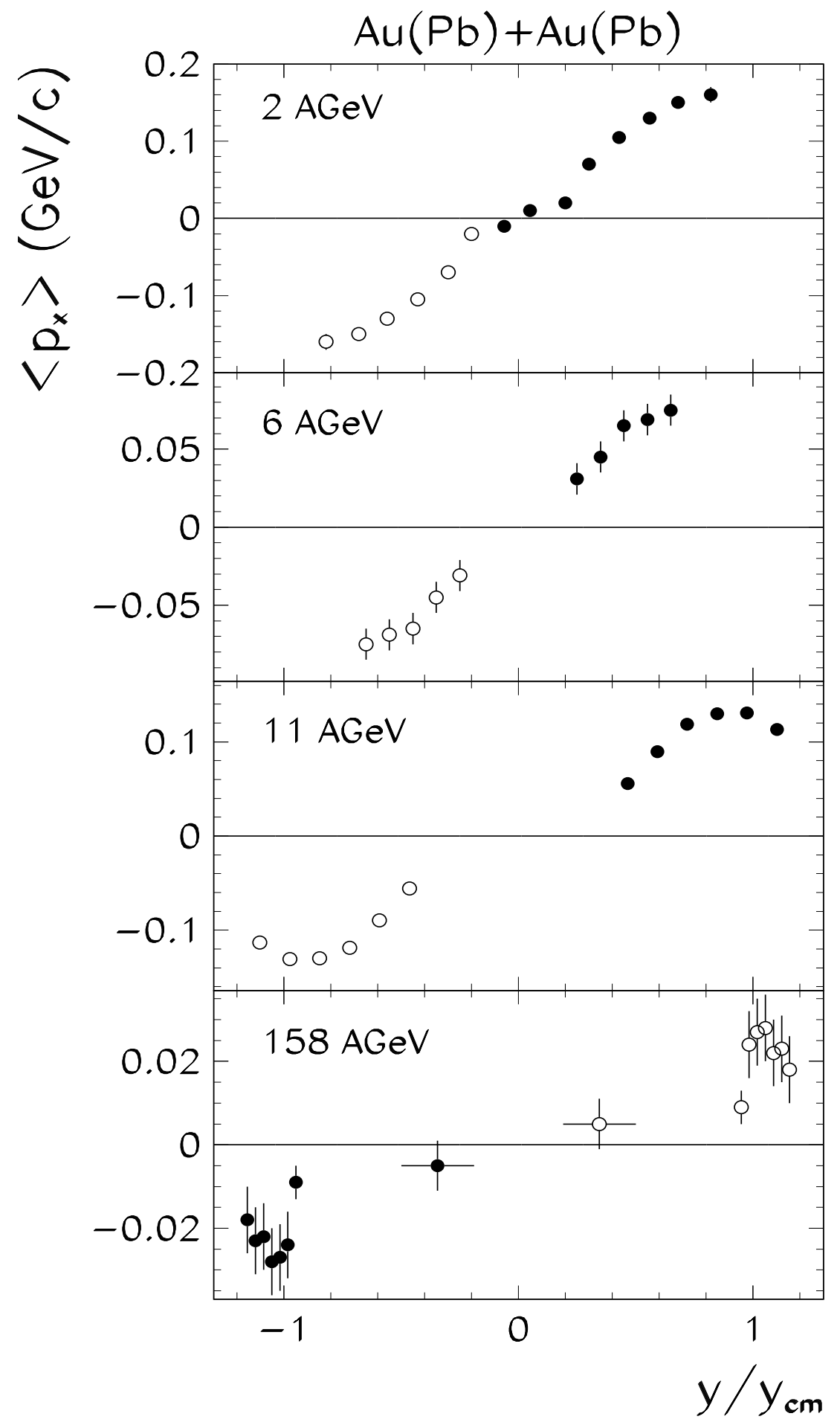

Fig. 2. Results of transverse flow analyses, $P_{x}$ vs. $y / y_{c m},[8,8,10,12]$. $158 \mathrm{AGeV}$ results show strong softening. 


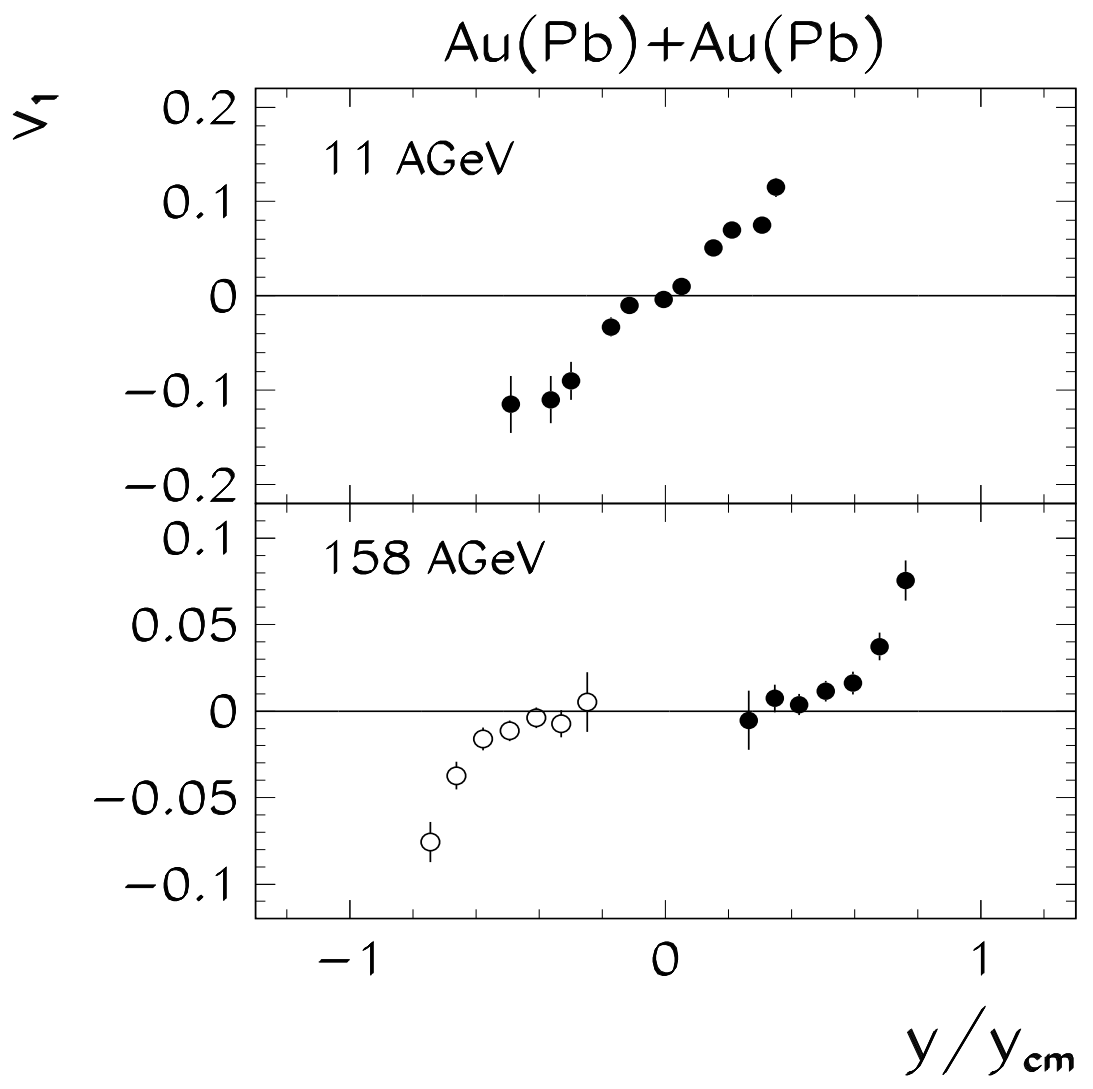

Fig. 3. Results of azimuthal Fourier analyses of directed flow, $v_{1}$ vs. $y / y_{\mathrm{cm}}[10,11]$. Low energy (below $2 \mathrm{AGeV}$ ) data show no CM softening in neither $P_{x}$ or $v_{1}$ analyses. 158 A.GeV $\mathrm{Pb}+\mathrm{Pb}$ data show obvious and strong softening, $\sim 100 \%$. 